\title{
Possible new phase of thermal QCD
}

\author{
Andrei Alexandru, ${ }^{1, *}$ and Ivan Horváth ${ }^{2, \dagger}$ \\ ${ }^{1}$ The George Washington University, Washington, DC 20052, USA \\ ${ }^{2}$ University of Kentucky, Lexington, Kentucky 40506, USA
}

(Received 5 July 2019; revised manuscript received 10 October 2019; published 22 November 2019)

\begin{abstract}
Using lattice simulations, we show that there is a phase of thermal QCD, where the spectral density $\rho(\lambda)$ of the Dirac operator changes as $1 / \lambda$ for infrared eigenvalues $\lambda<T$. This behavior persists over the entire low energy band we can resolve accurately, over 3 orders of magnitude on our largest volumes. We propose that in this "IR phase," the well-known noninteracting scale invariance at very short distances (UV, $\lambda \rightarrow \infty$, asymptotic freedom), coexists with a very different interacting type of scale invariance at long distances (IR, $\lambda<T)$. Such dynamics may be responsible for the unusual fluidity properties of the medium observed at RHIC and LHC. We point out its connection to the physics of the Banks-Zaks fixed point, leading to the possibility of massless glueballs in the fluid. Our results lead to the classification of thermal QCD phases in terms of IR scale invariance. The ensuing picture naturally subsumes the standard chiral crossover feature at " $T_{c}$ " $\approx 155 \mathrm{MeV}$. Its crucial new aspect is the existence of temperature $T_{\mathrm{IR}}\left(200 \mathrm{MeV}<T_{\mathrm{IR}}<250 \mathrm{MeV}\right)$ marking the onset of the IR phase and possibly a true phase transition.
\end{abstract}

DOI: $10.1103 /$ PhysRevD.100.094507

\section{INTRODUCTION}

The study of strongly interacting matter as a function of temperature and baryon density is an active area of theoretical and experimental research (see [1] for recent review). At high energies of colliding heavy nuclei, such as those studied at LHC and the high end of RHIC, baryon densities are small enough so that the results are generally expected, among other things, to shed light on the nature and properties of thermal QCD transition in the early universe. In this regime, it has become widely accepted, largely due to the matured power of lattice QCD [2], that increasing temperature leads to a smooth crossover in properties of thermal strongly interacting matter. On the experimental side, results from RHIC [3-6] and LHC [7] based on modeling the time evolution of collisions in terms of relativistic hydrodynamics, produced a picture of a strongly coupled liquidlike medium with extremely low $\eta / s$ (shear viscosity/entropy density) at high temperatures. In parallel and initially independent developments, similar values of $\eta / s$ were obtained in highly symmetric and strongly coupled gauge theories with a large number of colors, studied by means of their holographic dual [8].

\footnotetext{
*aalexan@gwu.edu

ihorv2@g.uky.edu
}

Published by the American Physical Society under the terms of the Creative Commons Attribution 4.0 International license. Further distribution of this work must maintain attribution to the author(s) and the published article's title, journal citation, and DOI. Funded by SCOAP.
This sparked a flurry of attempts to model the medium seen in the experiments via more refined descriptions of this type.

However, the physics of thermal QCD transition(s) and the nature of the discovered liquidlike state of matter are far from settled, even in the limit of vanishing net baryon density $(\mu=0)$, the setting of our interest. Among other things, the currently favored scenario involving a single feature (crossover at " $T_{c}$ ") offers limited room for accommodating a dramatic change from a medium described as a weakly interacting hadron resonance gas to a strongly interacting near-perfect fluid. In this work, we propose a hierarchy of thermal effects in QCD, based on scale invariance properties at long distances, which adds new detail to the existing picture, and ties with it in a natural manner. A special role in our analysis will be played by glue fields. In fact, one of our conclusions is that, from the standpoint of scale invariance, the phase structure of pure glue $\mathrm{SU}(3)$ gauge theory (pgQCD) and that of nature's strong interactions (QCD) are qualitatively the same. Avoiding the complication of quark fields, we thus first describe the proposed picture in the context of the former.

Since pgQCD is a theory of massless vector fields, it is classically scale invariant. According to the standard picture at zero temperature, this scale invariance is broken by quantum effects, leading to a low energy scale and the spectrum of massive bound states (glueballs). Yet, at asymptotically short distances, the system can be effectively described by perturbing noninteracting gluons (asymptotic freedom $[9,10]$ ). This is sometimes rephrased as scale invariance being broken at long distances (IR), 


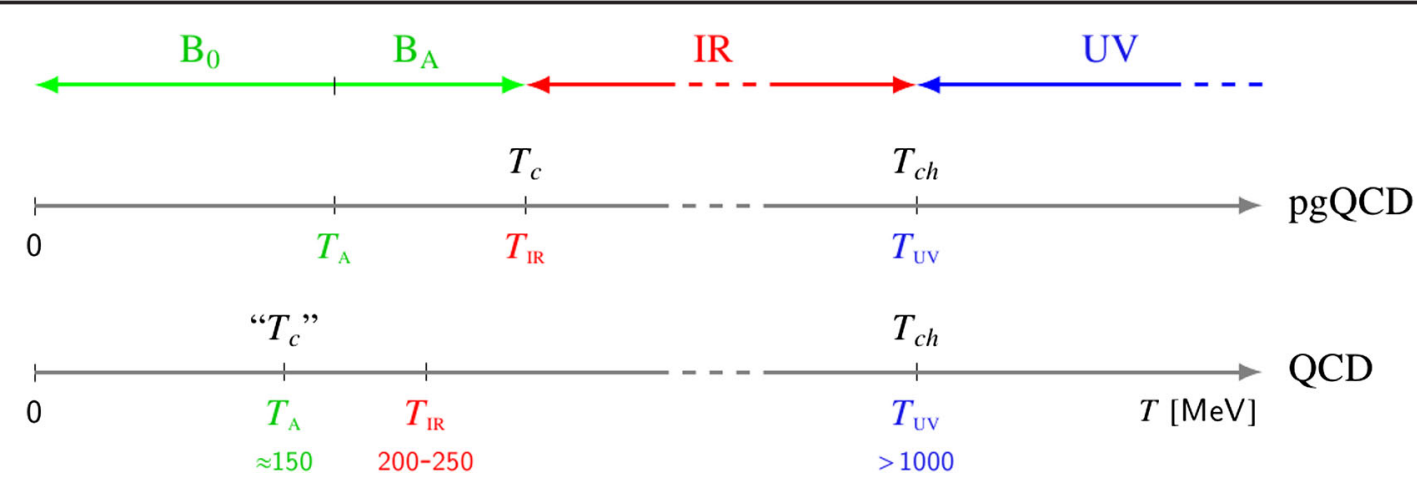

FIG. 1. Common thermal phase structure of pgQCD and QCD in terms of scale invariance. Since pgQCD is but a model of QCD glue, setting its physical scales involves small arbitrariness. Temperatures in black appeared in literature without reference to scale invariance.

but present at asymptotically short distances (UV) in the trivial noninteracting form.

Here we propose and support the following behavior of thermal pgQCD. Turning the temperature gradually on, the scale (non)invariance properties of a thermal state remain similar to that of a zero-temperature vacuum, until the scale of thermal agitation becomes comparable to the lowest scale of broken scale invariance ("gluon condensate"). This is characterized by the crossover temperature $T_{\mathrm{A}}$ past which the properties of the thermal medium change rapidly toward the restoration of scale invariance in IR. The latter then occurs at a well-defined temperature $T_{\mathrm{IR}}>T_{\mathrm{A}}$. In the ensuing range $T_{\mathrm{IR}}<T<T_{\mathrm{UV}}$ (IR phase), gauge fields characteristic of a thermal state are scale invariant at distances larger than $\approx 1 / T$. Unlike asymptotic scale invariance in UV, present at all temperatures, IR invariance emerges due to interaction that is still strong at long distances. For $T>T_{\mathrm{UV}}$ (UV phase), the field fluctuations in the IR regime $(\lambda<T)$ effectively disappear, and the notion of IR scale invariance becomes trivial. The system can then be described as weakly interacting gluon plasma.

This scenario is schematically shown in Fig. 1 (top, middle). Note that the low-temperature region $T<T_{\mathrm{IR}}$ (B phase for "broken") is split into two regimes $\mathrm{B}_{0}$ and $\mathrm{B}_{\mathrm{A}}$ by $T_{\mathrm{A}}$. The relation to transition temperatures discussed previously in the literature without invoking scale invariance is also indicated. Temperature $T_{\mathrm{IR}}$ coincides with the well-known $T_{c}$ of Polyakov line first order transition in pgQCD [11]. In addition, we identify $T_{\mathrm{UV}}$ with $T_{\mathrm{ch}}$ of chiral polarization transition [12-14]. Analog of $T_{\mathrm{A}}$ has not appeared in the context of pgQCD. ${ }^{1}$

Next, we present evidence that the above $T$ pattern of scale invariance in a gauge field is retained by QCD of nature (Fig. 1, bottom). In other words, scaling properties of QCD glue, which again enters as a nominally scale-free

\footnotetext{
${ }^{1}$ Transition with analogous physical meaning was in fact discussed in Ref. [14] but, rather than being attributed to a distinct dynamical effect, it was mistakenly identified with $T_{c}$ in pgQCD.
}

entity, are driven by quantum nature of the theory like in pgQCD, rather than quark mass effects. Thus, there is a crossover temperature $T_{\mathrm{A}} \approx 150 \mathrm{MeV}$ which we qualitatively associate with chiral " $T_{c}$ " of the standard scenario. However, here it is simply a characteristic temperature of the B phase, marking the onset of changes toward IR scale invariance. Note that the quark condensate now also plays a role in determining the value of $T_{\mathrm{A}}$. The IR phase then emerges at $200<T_{\mathrm{IR}}<250 \mathrm{MeV}$.

Before proceeding to lattice evidence, we address several immediate questions.

(i) Since lattice offers good quantitative control over QCD at $\mu=0$, how did the IR phase escape the detection? The answer is insufficient volumes. Indeed, the usual expectation is that IR scales $\Lambda<T$ contribute little to physics for $T>$ " $T_{c}$ ". Our proposal not only contradicts this but implies that, for $T_{\mathrm{IR}}<T<T_{\mathrm{UV}}$, it is the deep infrared scales $\Lambda \ll T_{\mathrm{IR}}$ that drive a significant IR contribution. Hence, we predict the existence of a "crossover size" $L_{\mathrm{IR}}(T) \gg 1 / T_{\mathrm{IR}}$ of the system past which the deep infrared physics becomes readily reflected in thermal observables. The systems of sufficiently large spatial sizes $L>L_{\mathrm{IR}}(T)$ are not commonly studied at present. This is expanded upon in Appendix A.

(ii) Given (i), how is the existence of the IR phase inferred from lattice simulations? At $T \gtrsim T_{\mathrm{IR}}$, we detect the onset of scale invariant $1 / \lambda$ behavior of Dirac spectral density $\rho(\lambda)$ (number of eigenmodes per unit volume and spectral interval) for $\lambda \lesssim T$. We propose that this arises due to the onset of effective IR scale invariance of glue fields dominating the thermal state. ${ }^{2}$ While the two notions are not equivalent a priori, they are consistent (Appendix B). Moreover, in theories with IR scale invariant gauge fields, such as those governed by Banks-Zaks fixed point, the pure power law behavior of $\rho(\lambda)$ is expected due to its

\footnotetext{
${ }^{2}$ The strict claim $\rho(\lambda) \propto 1 / \lambda$ for $\lambda \lesssim T$ seemingly entails integrability issues, but these are superficial in light of regularizations involved. For this discussion, one can simply assume $\rho(\lambda) \propto 1 / \lambda^{1-\delta}, 0<\delta \ll 1$.
} 

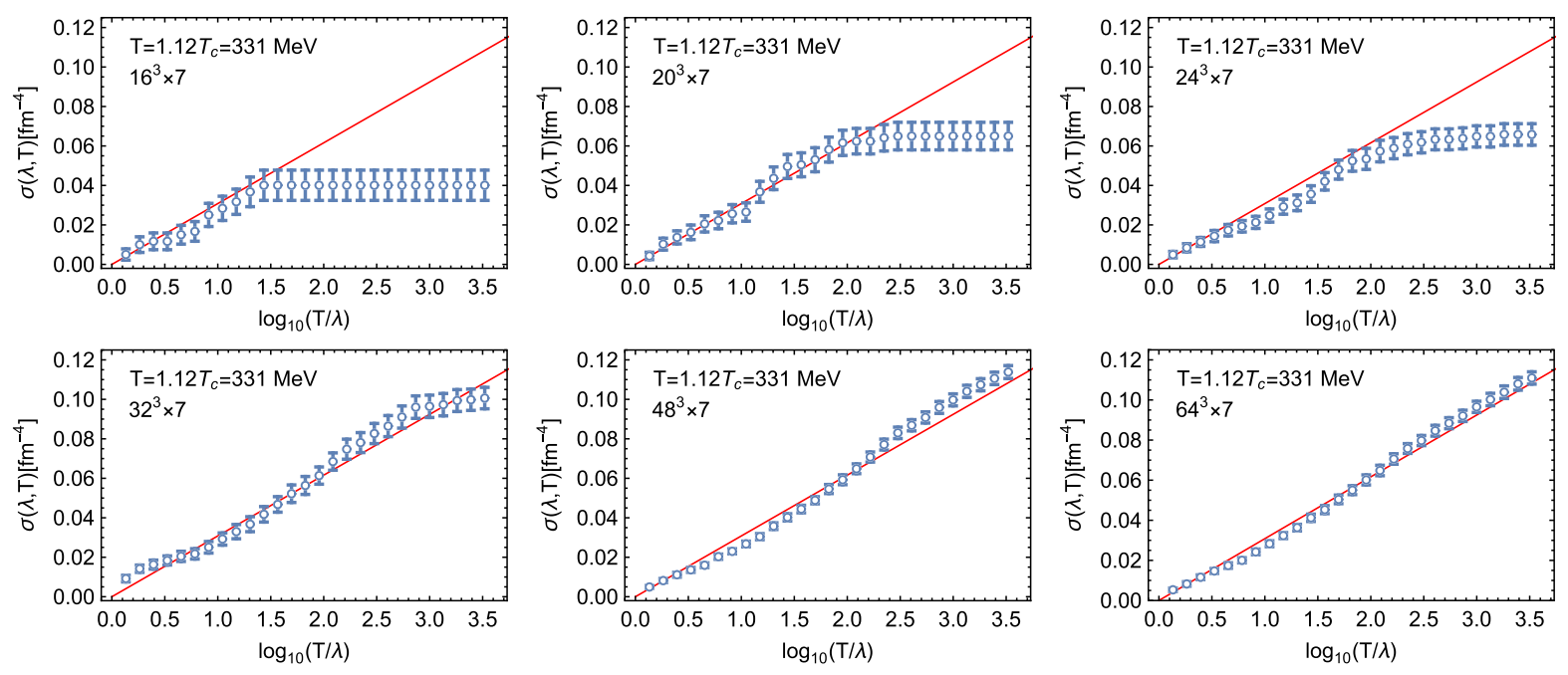

FIG. 2. IR dependence $\rho(\lambda) \propto 1 / \lambda$ emerging in pgQCD at $T=1.12 T_{c}$ and UV cutoff $a=0.085 \mathrm{fm}$.

proposed connection to mass anomalous dimension $[15,16] .^{3}$ This argument also suggests that, up to small quark mass deformations, IR scale invariance of glue extends to the quark sector in QCD, which was implicitly assumed already.

(iii) Scale invariance in field theory is normally addressed via the energy-momentum tensor. However, such test should only include scales up to $\Lambda_{\mathrm{IR}}(T) \lesssim T$ (upper edge of $1 / \lambda$ ) in this case. In conjunction with (i), this avoids the conflict with existing lattice results $[17,18]$.

(iv) Given its perturbative nature, the UV phase should only ensue when thermal agitation mostly engages perturbative scales. In that vein, our expectation is that $T_{\mathrm{UV}}>1 \mathrm{GeV}$ (Fig. 1). Its precise determination in lattice simulations is challenging in part because the minimal system size needed to detect the IR phase grows with temperature (Appendix A).

\section{LATTICE EVIDENCE}

Technical details of our simulations are summarized in Appendix C. To discuss the results, we start with pgQCD where needed volumes are more readily accessible. In Ref. [19], a peak at the infrared end of Euclidean Dirac spectral density has been observed in pgQCD above $T_{c}$. Only recently it was shown [14] that this feature is not a regularization artifact. Here we present evidence that $\rho(\lambda) \propto \lambda^{-1}$ in IR which, together with $\rho(\lambda) \propto \lambda^{3}$ in UV, generates a bimodal structure facilitating scale invariance at both ends of the spectrum.

To that end, we study the spectrum of the overlap Dirac operator on equilibrium backgrounds. A useful quantifier is the volume density of eigenmodes in spectral range $[\lambda, T]$, namely

\footnotetext{
${ }^{3}$ Note that $\rho(\lambda) \propto 1 / \lambda^{1-\delta}, 0<\delta \ll 1$ would imply very large anomalous dimensions in those theories.
}

$\sigma(\lambda, T) \equiv \int_{\lambda}^{T} \rho(\omega) d \omega \rightarrow c(T) \ln \frac{T}{\lambda} \quad$ for $\rho(\lambda)=\frac{c}{\lambda}$.

If $\rho(\lambda) \propto \lambda^{-1}$ for $\lambda<T$, a straight line passing through the origin is obtained in variable $x=\ln T / \lambda \geq 0$. Note that $\lambda=T$ corresponds to $x=0$ and IR is approached by increasing $x$. If $\rho(\lambda) \propto \lambda^{-1}$ only for $\lambda<\Lambda_{\mathrm{IR}}(T)<T$, a $y$-shifted linear segment appears for $x>\ln T / \Lambda_{\mathrm{IR}}$.

In Fig. 2 we show $\sigma(x)$ in pgQCD on increasing volumes (UV cutoff $a=0.085 \mathrm{fm}$ ) at $T=1.12 T_{c}$. Each case involves an easily identifiable, approximately linear segment extending from origin to increasingly IR scales as the IR cutoff $L$ increases. Leveling off at larger $x$ signals the IR edge of the spectrum. On the largest volume $(L=5.4 \mathrm{fm})$, the $1 / \lambda$ behavior persists over 3.5 orders of magnitude from $T$ down to deep infrared. The eye-guiding red line is the same in each plot and corresponds to the fit for largest volume, producing the value $c\left(1.12 T_{c}\right)=0.0308(3) \mathrm{fm}^{-4}$ in Eq. (1). In Appendix D, we discuss a more direct approach to exposing the $1 / \lambda$ dependence of $\rho(\lambda)$ over a wide range of scales.

To assess the relationship of Polyakov line phase transition in pgQCD to its IR phase, we simulate the system at $T=0.98 T_{c}$ in the otherwise identical setup with large volume. The resulting $\rho(\lambda)$ is shown in Fig. 3 (top left). Apart from saturation at the IR edge of the spectrum, we find no linear segment in the corresponding $\sigma(x)$ (top middle), in direct contrast to $1 / \lambda$ behavior at $T=1.12 T_{c}$ (top right). Thus, barely below $T_{c}$, the system is in the $\mathrm{B}$ phase. Note also the characteristic difference in spectral densities between B and IR phases (top left). Given the above and the corroborating spectral evidence of Ref. [14] at $T=1.02 T_{c}$, we conclude that $T_{\mathrm{IR}}$ coincides with $T_{c}$. One consequence of this is that the $\mathrm{B}$ and IR phase of pgQCD are separated by a first order phase transition. 

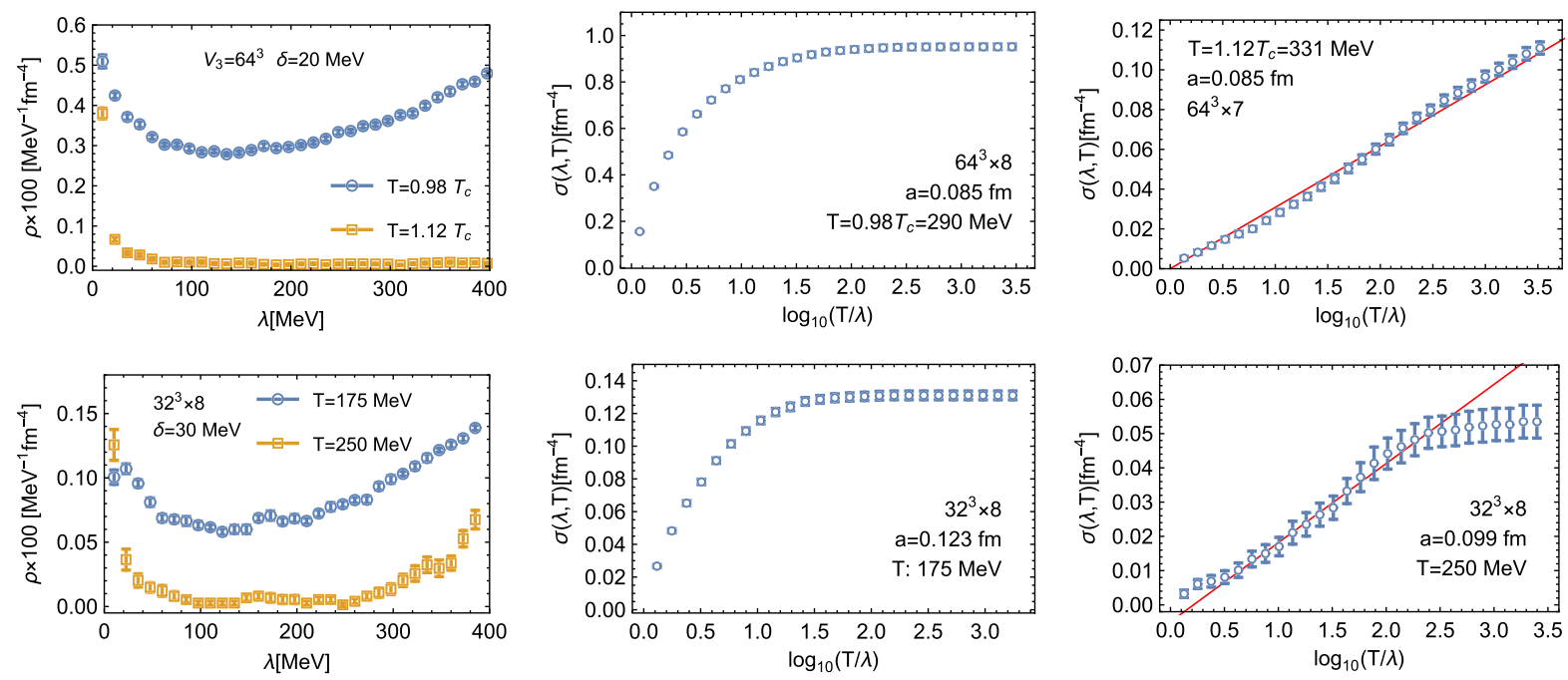

FIG. 3. Thermal transition to IR phase in pure glue QCD (top) and QCD (bottom).

An important feature of the Dirac spectrum at $T=$ $0.98 T_{c}$ is that $\rho(\lambda)$ exhibits the IR peak even at $T<T_{\mathrm{IR}}$. Indeed, there is a minimum of $\rho(\lambda)$ at $\lambda_{m} \approx 120 \mathrm{MeV}$ (Fig. 3, top left). Such minimum at $\lambda_{m}>0$ may exist even at zero temperature due to the possible logarithmic divergence at $\lambda \rightarrow 0$ and/or the presence of positive power with negative prefactor [20]. However, this has not yet been confirmed in pgQCD simulations, implying that $\lambda_{m}(T)$ is very small or zero at low $T$. This leads us to propose that it is meaningful to distinguish the $T \gtrsim 0$ and $T \lesssim T_{\text {IR }}$ regimes by a crossover characterized by temperature $0<T_{\mathrm{A}}<T_{\mathrm{IR}}$. While the crossover point is a nonunique concept, here we have in mind a commonly used approach based on the rate of change. In other words, we define $T_{\mathrm{A}}$ as the position of maximum (peak) in $d \lambda_{m} / d T$. In physics terms, $T_{\mathrm{A}}$ relates to the point at which the gluon condensate becomes significantly affected by thermal agitation. It splits the $\mathrm{B}$ phase into regimes $\mathrm{B}_{0}$ and $\mathrm{B}_{\mathrm{A}}$ (Fig. 1) with the latter referred to as anomalous, conforming to terminology of Ref. [14].

Standard expectations suggest that the IR phase, commencing at $T_{\mathrm{IR}}$, ends at temperature $T_{\mathrm{UV}}$ (Fig. 1) above which scales $\lambda \approx T$ become amenable to perturbative treatment. Since the IR peak is not featured in the weakly coupled regime, we define $T_{\mathrm{UV}}$ as a temperature at which $\rho(\lambda)$ becomes a nondecreasing function on $\lambda \geq 0$ with $\rho(0)=0$. The associated disappearance of the IR peak has been observed on moderate volumes in Refs. [12,13], accompanied by the simultaneous loss of chiral polarization in low-lying Dirac modes. Since the latter effect is characterized by temperature $T_{\mathrm{ch}}$, we propose that $T_{\mathrm{UV}}=T_{\mathrm{ch}}$ as indicated in Fig. 1 .

We now turn to overlap spectral densities in QCD. More specifically, we study SU(3) gauge theory with $N_{f}=2+1$ quark flavors at physical masses (see Appendix C), which is a very precise representation of real-world strong interactions. To support the existence of $T_{\mathrm{IR}}$, we show in
Fig. 3 (bottom) the analog of the IR transition we described in pgQCD. The clearly noninvariant behavior at $T=$ $175 \mathrm{MeV}$ is contrasted with that at $T=250 \mathrm{MeV}$. The latter exhibits characteristic features of the IR phase, both in terms of $\rho(\lambda)$ and $\sigma(x)$. Regarding the latter, note also the similarity to the pattern displayed by volume sequence in Fig. 2. In Appendix D we present additional results at $T=200 \mathrm{MeV}$, featuring the behavior more marginally on the $\mathrm{B}_{\mathrm{A}}$ side. This leads us to the following initial estimates:

$200 \mathrm{MeV}<T_{\mathrm{IR}}<250 \mathrm{MeV}, \quad T_{\mathrm{A}} \approx 150 \mathrm{MeV} \lesssim “ T_{c}$ ”

where " $T_{c}$ " is the temperature of chiral crossover. The estimate of $T_{\mathrm{A}}$ follows from our analysis in Ref. [14] (Fig. 5 of that work). There it was found that $\lambda_{m}$ becomes essentially undetectable at $T=150 \mathrm{MeV}$ (simulated size $L=5.3 \mathrm{fm}$ ) signaling either its significant drop at lower temperatures, so that significantly larger volumes are needed to reveal it, or its entire disappearance $\left(\lambda_{m} \rightarrow 0\right)$. Both options entail the proximity of a dividing point between $\mathrm{B}_{0}$ and $\mathrm{B}_{\mathrm{A}}$ regimes, namely $T_{\mathrm{A}}$.

\section{IR-UV SEPARATION AND BANKS-ZAKS FIXED POINT}

The signature aspect of transition at $T_{\mathrm{IR}}$ is a clean separation of IR and UV scales in the gauge field, reflected by almost perfectly bimodal $\rho(\lambda)$. Additional data illustrating the latter is presented in Appendix D. The analysis of Refs. [13,14,21,22] revealed that, apart from increasing the temperature, such IR-UV separation is also inducible by decreasing the quark mass or increasing the number of flavors in parameter space $\left(T, m_{i}, N_{f}\right)$ of $\mathrm{SU}(3)$ gauge theories with fundamental quarks. Our aim is to integrate the new element of IR scale invariance into these findings, 
which promises a valuable insight into the nature of the IR phase in thermal QCD.

We start in the corner of SU(3) theory space which is native to IR scale invariance, namely the vicinity of the conformal window [23] ( $N_{f}$ massless flavors, $N_{f}^{c}<N_{f}<$ $16.5, T=0)$. In Refs. $[13,14,21,22]$ it was found that small mass dynamics at $N_{f}=12$, believed by most researchers to be near conformal, generates the pattern of IR-UV separation closely mimicking that of QCD in the IR phase. While originally interpreted as indicating an unexpectedly large $N_{f}^{c}$, the revelation that $\rho(\lambda)$ may be a pure power in IR begs this to be reconsidered since $\rho(\lambda) \propto \lambda^{p}$ is exactly what one expects near conformality. A consistent inference is the split of the conformal window into a strongly coupled part $N_{f}^{c} \equiv N_{f}^{\mathrm{IR}}<N_{f}<N_{f}^{\mathrm{UV}}$ with $p<0$, and the weakly coupled part $N_{f}^{\mathrm{UV}}<N_{f} \leq 16.5$ with $p>0$. The parametric trends in IR-UV separation then lead us to propose that strongly coupled regimes $T_{\mathrm{IR}}<T<T_{\mathrm{UV}}$ of $\mathrm{QCD}$ and $N_{f}^{\mathrm{IR}}<N_{f}<N_{f}^{\mathrm{UV}}$ of the conformal window belong to a single contiguous IR phase in SU(3) theory space, defined by $p<0$. In this sense, the observed elements of IR scale invariance in thermal QCD descend from conformality of a strongly coupled Banks-Zaks fixed point.

The above argument introduces an unconventional scenario for dynamics in a strongly coupled conformal window which requires more detail. Consider the $T=0$ system at $N_{f}^{\mathrm{IR}}<N_{f}<N_{f}^{\mathrm{UV}}$ with varying degenerate quark mass $m$. At large $m$, glue behaves as in pgQCD: there is no scale invariance in IR and the gauge coupling runs indefinitely. At asymptotically small $m$, the running stops at dynamically generated $\Lambda_{\mathrm{IR}}>0$, and scale invariance ensues at larger distances: the interacting fixed point entirely runs the IR, while the Gaussian one governs UV. The evidence of Refs. [13,14,21,22] suggests that the Banks-Zaks fixed point turns on in a sudden manner by theory entering the IR phase $\left(m<m_{\mathrm{IR}}\right)$ in mass. In more detail, the sequence

$$
m=\infty \stackrel{\mathrm{B}_{0}}{\longrightarrow} m_{\mathrm{A}} \stackrel{\mathrm{B}_{\mathrm{A}}}{\longrightarrow} m_{\mathrm{IR}} \stackrel{\mathrm{IR}}{\longrightarrow} 0
$$

produces B and IR phases as in the thermal case. With $N_{f}$ in a strongly coupled regime, the UV phase does not materialize. Thus, the mass vicinity of theory in a strongly coupled conformal window (its IR phase) is characterized by $\rho(\lambda) \propto \lambda^{p}$ where ${ }^{4}$

$$
\begin{aligned}
& p<0, \quad 0<m<m_{\mathrm{IR}}, \\
& 0<\lambda<\Lambda_{\mathrm{IR}}, \quad \lim _{m \rightarrow 0} \Lambda_{\mathrm{IR}}(m)>0 .
\end{aligned}
$$

\footnotetext{
${ }^{4}$ Note that conformality constraints on unitarity [24] and the conjectured method [15] of extracting $\bar{\psi} \psi$ anomalous dimensions then raise interesting questions on details of the $m \rightarrow 0$ limit and its relation to $m \equiv 0$
}

Among appealing features of the above is that it naturally explains the generation of $\Lambda_{\mathrm{IR}}$. Indeed, the degree of IR-UV separation reflects the extent to which IR and UV fixed points in Wilson theory space dominate the dynamics of the given field theory in respective scale domains. The nearly perfect scale separation in the IR phase suggests that these domains are described by almost decoupled IR and UV subtheories. Since $\Lambda_{\mathrm{IR}}$ defines the domain of IR, integrating out UV degrees of freedom (UV theory) only feeds into the dynamics of IR (IR theory) in a trivial manner. This stops the running since IR theory is scale invariant.

To see the relevance of this connection, consider the $m \rightarrow 0$ limit in SU(3) theory space. ${ }^{5}$ For $N_{f}=2$ (chiral $\mathrm{QCD})$ at $T=0$, this produces a massive physical spectrum except for Goldstone pions. In contrast, for $N_{f}^{\mathrm{IR}}<N_{f}<$ $N_{f}^{\mathrm{UV}}$ it leads to massless behavior in all channels due to the approached conformality. Since the property of infinite correlation length is stable within the contiguous IR phase, ${ }^{6}$ it extends from the strongly coupled conformal regime $\left(N_{f}^{\mathrm{IR}}<N_{f}<N_{f}^{\mathrm{UV}}, T=0\right)$ to the IR regime of chiral QCD $\left(N_{f}=2, T_{\mathrm{IR}}<T<T_{\mathrm{UV}}\right)$. Our approach thus predicts that the lowest "screening masses" (spatial correlations) and "quasiparticle masses" (timelike correlations) are zero in the IR phase of chiral QCD. Note that we do not claim the absence of excitations with masses larger than $T$. In fact, additive contributions to correlators by masses larger than $\Lambda_{\mathrm{IR}}\left(T, N_{f}\right)$ distinguish the low energy scale invariance of the IR phase from strict conformality present only at $T=0$.

Strong interactions of nature turn on small $m$ at $N_{f}=2$, but the above picture of the IR phase only gets corrected by light lowest masses in meson and baryon correlation functions. The IR scale invariance of glue, inherent to the IR phase, is expected to keep correlators of glue operators long range, and the associated glueball-like excitations massless.

\section{SYNTHESIS AND MAIN POINTS}

We proposed the existence of a new phase in thermal QCD, the IR phase $T_{\mathrm{IR}}<T<T_{\mathrm{UV}}$, featuring aspects of scale invariance at distances larger than $1 / \Lambda_{\mathrm{IR}}$, where $\Lambda_{\mathrm{IR}}(T) \lesssim T$. In particular, our way of probing the system suggests that glue fields dominating the thermal state in the IR phase are statistically self-similar upon rescalings involving such distances (Appendix B).

In the standard scenario, QCD matter enters the nearperfect fluid regime above the chiral crossover temperature " $T_{c} " \approx 155 \mathrm{MeV}$. However, given that scale invariance underlies model descriptions able to mimic the observed fluidity properties [8], we propose that this transition

\footnotetext{
${ }^{5}$ We refer to theories defined as $\lim _{m \rightarrow 0} \lim _{L \rightarrow \infty}$ of those with $N_{f_{6}}$ mass-degenerate flavors.

${ }^{6}$ Due to the above monotonicity properties, the IR phase in this restriction remains contiguous.
} 
actually occurs at $T_{\mathrm{IR}}\left(200<T_{\mathrm{IR}}<250 \mathrm{MeV}\right)$. In other words, the strongly interacting near-perfect fluid is realized by the IR phase. If glue fields continue to follow the described patterns arbitrarily deep into IR, then $T_{\mathrm{IR}}$ marks a phase transition where the leading IR power in $\rho(\lambda)$ changes from $p=0$ to $p \gtrsim-1$. This transition could be consequential for the analysis of heavy ion experiments and for modeling the thermal history of the universe.

The observed elements of IR scale invariance can be understood by viewing thermal QCD in the larger context of asymptotically free SU(3) gauge theories with fundamental quarks. To that end, we proposed the phase structure in this space that can be summarized by

$$
\text { phase }=\left\{\begin{array}{ll}
\mathrm{B} & \text { if } p=0 \\
\mathrm{IR} & \text { if } p<0 \\
\mathrm{UV} & \text { if } p>0
\end{array} \quad \text { where } \rho(\lambda) \propto \lambda^{p} \quad \text { for } \lambda \rightarrow 0\right.
$$

with transitions occurring accordingly. For example, increasing the temperature past $T_{\mathrm{IR}}$ in QCD is expected to eventually generate a transition from $p \gtrsim-1$ to $p>1$, identifying $T_{\mathrm{UV}}{ }^{7}$ The connection to scale invariance stems from the proposed existence of a contiguous IR phase in $\mathrm{SU}(3)$ space, in which UV field fluctuations associated with Gaussian fixed point $\left(A^{\mathrm{UV}}, \rho \propto \lambda^{3}\right)$ coexist with IR fluctuations driven by strongly coupled Banks-Zaks fixed point $\left(A^{\mathrm{IR}}, \rho \propto \lambda^{-1+\delta}\right)$. The high $T\left(T_{\mathrm{IR}}<T<T_{\mathrm{UV}}\right)$ medium of QCD (near-perfect fluid) and low $T(T \gtrsim 0)$ medium of strongly coupled near-conformal theory $\left(N_{f}^{\mathrm{IR}}<\right.$ $N_{f}<N_{f}^{\mathrm{UV}}$ ) both belong to this phase and thus share important dynamical features. This may prove useful in guiding the analytic attempts to describe the nearperfect fluid.

The conjecture that the IR phase of QCD realizes the near-perfect fluid is expected to have phenomenological consequences. For example, using the above connection to the physics of a strongly coupled conformal window, we have argued for a highly unusual spectrum of excitations (quasiparticles and screening masses) in the fluid. The fluctuations originating from a strongly coupled BanksZaks fixed point generate a narrow IR band of massless (glueball-like) and light (hadronlike) excitations. At the same time, fluctuations tied to Gaussian fixed point produce excitations in the UV band, creating a large gap $(>T)$. We hypothesize that the remarkable IR-UV separation, both in terms of fluctuating quantum fields and the resulting physical excitations, is one of the key

\footnotetext{
${ }^{7}$ The value of $p$ in a thermal UV phase could be infinite if the depletion of modes in the infrared proceeds faster than arbitrary positive power, e.g., if a gap develops in the Dirac spectrum. Note also that $p=0$ (B phase) includes the case of logarithmically diverging density.
}

ingredients driving the exotic properties of the strongly coupled medium discovered at the RHIC and LHC.

The central point of this work, namely the existence of new infrared dynamics in gauge theories (IR phase), invites additional corroboration and further clarification. For example, a large scale study confirming $\rho(\lambda) \propto 1 / \lambda$ in QCD at the level comparable to pgQCD (Fig. 2) is of primary interest. Similar quantitative analyses in the vicinity of a strongly coupled conformal window (e.g., $N_{f}=12$ ) are also very desirable. Studies examining the proposed association of power law Dirac densities with scale invariant gauge fields by direct means would solidify and refine the interpretation of the IR phase. In the process, such works may also clarify why $1 / \lambda$ (rather than other power) emerges in QCD.

\section{ACKNOWLEDGMENTS}

A. A. is supported in part by the National Science Foundation CAREER Grant No. PHY-1151648 and by U.S. DOE Grant No. DE-FG02-95ER40907. I. H. acknowledges support from the Department of Anesthesiology at the University of Kentucky, help from Jian Liang and Robert Mendris, as well as conversations with Anatoly Dymarsky and Ganpathy Murthy. We thank Wuppertal-Budapest collaboration for sharing their lattice ensembles.

Note added.-While this manuscript was in preparation, work [25] appeared, suggesting a different new feature in thermal QCD based on chiral considerations.

\section{APPENDIX A: THE CROSSOVER SIZE}

The IR phase of QCD is characterized by $\rho(\lambda) \propto 1 / \lambda$ for $\lambda<\Lambda_{\mathrm{IR}}$. Here $\Lambda_{\mathrm{IR}}$ determines the minimal distance over which scale invariance of glue fields applies. But what is the minimal size of the system in which this behavior affects an observable in a discernible manner? This role is played by the "crossover size" $L_{\mathrm{IR}}(\mathrm{Sec} . \mathrm{I}){ }^{8}{ }^{8}$ Given that the IR contribution is driven by deep infrared $\left(\ll \Lambda_{\mathrm{IR}}\right)$ rather than the vicinity of $\Lambda_{\mathrm{IR}}$, it is clear that $L_{\mathrm{IR}} \gg 1 / \Lambda_{\mathrm{IR}}$ for standard observables. Moreover, the density of Dirac eigenmodes in an $\mathrm{R}$ regime drops quickly with temperature (see e.g., [13]), causing $L_{\mathrm{IR}}$ to increase. Sensitivity to scale invariant behavior of glue is then expected on systems of size $L$ satisfying

$L(T)>L_{\mathrm{IR}}(T)>L_{\mathrm{IR}}\left(T_{\mathrm{IR}}\right) \gg 1 / \Lambda_{\mathrm{IR}}\left(T_{\mathrm{IR}}\right) \gtrsim 1 / T_{\mathrm{IR}}$

where the last inequality is due to $\Lambda_{\mathrm{IR}}(T) \lesssim T$. Hence, $L(T) \gg 1 / T_{\mathrm{IR}}$ applies to all standard observables and all temperatures $T_{\mathrm{IR}}<T<T_{\mathrm{UV}}$. Since $T_{\mathrm{IR}}$ is comparable

\footnotetext{
${ }^{8} L_{\mathrm{IR}}$ can be viewed as a size at which the associated finite volume correction assumes its asymptotic form.
} 
to $\Lambda_{\mathrm{QCD}}$, the relevant sizes are larger than typically considered sufficient for thermal QCD studies.

Lattice introduces a slight complication in that the Dirac operator, serving as the detector of IR scale invariance, is not a unique object: different discretizations capture aspects of continuum behavior to varying degrees. Chirality plays a relevant role here. Indeed, the bimodality in $\rho(\lambda)$ was first observed with an overlap operator that fully respects chirality, while it was not seen by the staggered operator on identical backgrounds [19]. However, the IR peak has recently been identified by a staggered-type operator in pgQCD on larger volumes [26], confirming that the presence of this feature is discretization independent. This is also consistent with bimodality of the overlap operator persisting into the continuum limit, shown in Ref. [14]. In addition, since $L_{\mathrm{IR}}$ has physical origin (see above), we expect that

$$
\lim _{a \rightarrow 0} L_{\mathrm{IR}}^{\mathrm{lat}_{1}}(T, a)=\lim _{a \rightarrow 0} L_{\mathrm{IR}}^{\mathrm{lat}_{2}}(T, a)=L_{\mathrm{IR}}(T)
$$

i.e., that $L_{\mathrm{IR}}(T)$ is universal for fixed definition of the crossover point.

In lattice QCD, a Dirac operator defining the quark part of the action obviously plays a special role. While the existence of an IR peak in this "native" Dirac spectrum appears more difficult to ascertain numerically, the studies focusing on the $\mathrm{U}_{A}(1)$ problem $[27,28]$ already suggest that the feature is present at physical light-quark masses, albeit the studied volumes are small. Its absence would in fact be very surprising. Indeed, the ensuing singularity in the space of lattice Dirac operators with respect to (A2), as well as the associated possibility of nonuniversality in topological susceptibility, make such a scenario unlikely.

\section{APPENDIX B: SCALE INVARIANCE AND DIRAC SPECTRAL DENSITY}

Our aim here is to illustrate how scale invariance of gauge field $A$ constrains the form of spectral density $\rho(\lambda \mid A)$ of the Euclidean Dirac operator $D=D[A]$. This is easiest to do in $\mathbb{R}^{4}$, the setup relevant for theories in a conformal window, but the arguments can be modified to finite temperature. We implicitly assume that $A_{\mu}(x) \in \operatorname{su}(N)$ although this is not important in the present context. Thus, we are dealing with the eigenvalue problem on a fixed "classical" background, defined by $\left[A_{\mu}(x)\right.$ is antiHermitian]

$$
D[A] \psi(x) \equiv \sum_{\mu} \gamma_{\mu}\left[\frac{\partial}{\partial x_{\mu}}-A_{\mu}(x)\right] \psi(x)=i \lambda \psi(x)
$$

where $\lambda \in \mathbb{R}$ and $\psi$ is an eigenmode. Let $A^{(s)}$ be the gauge field obtained from $A$ by the canonical scale transformation. The following are the simultaneous eigensystem triples:

$$
\begin{aligned}
(A, \psi, \lambda) & \leftrightarrow\left(A^{(s)}, \psi^{(s)}, s \lambda\right), \quad A^{(s)}(x) \equiv s A(s x), \\
\psi^{(s)} & \propto \psi(s x),
\end{aligned}
$$

where the correspondence is one to one. Envisioning the potentials singular at origin or infinity, we consider the regularized eigenvalue problem on $[\epsilon, L]^{4}$ with $\epsilon$ the ultraviolet and $L$ the infrared regulator. ${ }^{9}$ The relation (B2) is then modified as

$$
(A, \psi, \lambda, \epsilon, L) \leftrightarrow\left(A^{(s)}, \psi^{(s)}, s \lambda, \epsilon / s, L / s\right) .
$$

The standard (anti)periodic boundary conditions on $A, \psi$ are respected by the correspondence.

With the usual assumption that the spectrum on finite volume is discrete, (B3) implies that the number of eigenmodes in interval $\left[\lambda_{1}, \lambda_{2}\right]$ for setup on the left is the same as that in $\left[s \lambda_{1}, s \lambda_{2}\right]$ for setup on the right. Focusing on $A(x)$ with no singularity at $x \rightarrow \infty$ allows us to remove the infrared cutoff $(L \rightarrow \infty)$ and to account for the number of eigenmodes in terms of smooth spectral density. This then leads to

$$
\begin{aligned}
\int_{\lambda_{1}}^{\lambda_{2}} d \lambda \rho(\lambda \mid A, \epsilon) & =\frac{1}{s^{4}} \int_{s \lambda_{1}}^{s \lambda_{2}} d \lambda \rho\left(\lambda \mid A^{(s)}, \epsilon / s\right) \\
& =\frac{1}{s^{3}} \int_{\lambda_{1}}^{\lambda_{2}} d \lambda \rho\left(s \lambda \mid A^{(s)}, \epsilon / s\right)
\end{aligned}
$$

for all $\lambda_{1}$ and $\lambda_{2}$. Consequently,

$$
s^{3} \rho(\lambda \mid A, \epsilon)=\rho\left(s \lambda \mid A^{(s)}, \epsilon / s\right)
$$

which for scale invariant background $A^{(s)}(x)=A(x)$ leads to

$$
\rho(\lambda, \epsilon)=\lambda^{3} f(\lambda \epsilon)
$$

where $f(x)$ is an arbitrary non-negative function. Thus, for scale invariant free field $(A(x) \equiv 0)$, with no singularity at the origin, the density is $\epsilon$ independent and $\rho(\lambda) \propto \lambda^{3}$. However, no leading infrared power, such as the behavior $1 /\left(\lambda \epsilon^{4}\right)$, is excluded a priori. In quantum theory, the diverging UV cutoff length scale is replaced by the dynamically generated $1 / \Lambda_{\mathrm{IR}}$, and we thus have $\rho(\lambda) \propto$ $\Lambda_{\mathrm{IR}}^{4} / \lambda$. These considerations can be generalized to self-similar (rather than strictly scale invariant) gauge backgrounds, providing additional freedom to accommodate the $1 / \lambda$ dependence.

\footnotetext{
${ }^{9}$ The more symmetric setup on $([-L / 2,-\epsilon / 2] \cup[\epsilon / 2, L / 2])^{4}$ proceeds in an analogous way.
} 


\section{APPENDIX C: SUMMARY OF TECHNICAL DETAILS}

Our pgQCD simulations were performed using Wilson gauge action with a scale setting based on reference value $r_{0}=0.5 \mathrm{fm}$. The volume dependence of Dirac spectra was studied at $\beta=6.054$ which corresponds to UV cutoff $a=0.085 \mathrm{fm}$. The estimate of $T_{c}$ involved the results of Ref. [29]. The ensembles at $T=1.12 T_{c}\left(N_{\tau}=7\right)$ contain $400,400,400,200,200,100$ gauge configurations for $N=16,20,24,32,48,64$ systems, respectively $(1 / T=$ $N_{\tau} a$ and $\left.L=N a\right)$. The results at $T=0.98 T_{c}\left(N_{\tau}=8\right.$, $N=64)$ are based on 94 configurations.

For QCD Dirac spectra, we utilized the gauge ensembles of the Wuppertal-Budapest group described in Ref. [30]. More precisely, they were generated in $N_{f}=2+1$ theory at physical light quark mass of $\left(m_{u}+m_{d}\right) / 2$, and the physical "heavy" quark mass of $m_{s}$. In terms of lattice setup, the simulations used tree-level Symanzik-improved gauge action and stout-improved staggered fermions. The physical point (thus scale setting) was defined by fixing $m_{\pi}$, $m_{K}$, and $f_{K}$ to their physical values at zero temperature. Our analysis is based on 100 gauge configurations in each case.

The main object of our interest is the 4-volume density $\sigma\left(\lambda_{1}, \lambda_{2}\right)$ of Dirac eigenmodes from spectral interval $\left[\lambda_{1}, \lambda_{2}\right]$ [convention set by Eq. (B1)]. This quantity is commonly expressed in terms of the corresponding spectral density $\rho(\lambda)$, namely

$$
\sigma\left(\lambda_{1}, \lambda_{2}\right) \equiv \int_{\lambda_{1}}^{\lambda_{2}} d \lambda \rho(\lambda)
$$

Unless stated otherwise, exact zero modes are excluded from counting. On finite 4-volume $L^{3} / T$, the ensemble average is implicitly assumed in (C1), although expressing $\rho(\lambda)$ in terms of $\delta$ functions makes it meaningful even for single configuration.

In a numerical study, it is necessary to work with a coarse-grained version of $\rho(\lambda)$. This is achieved by introducing the parameter $\delta>0$ and defining

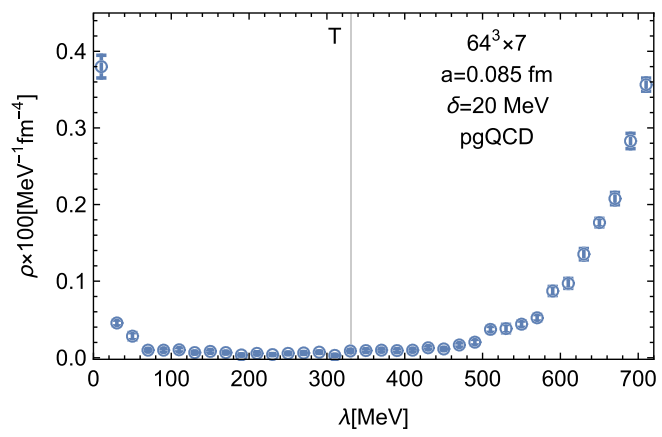

$$
\rho(\lambda, \delta) \equiv \frac{\sigma(\lambda-\delta / 2, \lambda+\delta / 2)}{\delta}, \rho(\lambda)=\lim _{\delta \rightarrow 0} \rho(\lambda, \delta) .
$$

Only $|\lambda|>\delta / 2+\epsilon$ with suitably chosen $\epsilon>0$ to avoid finite volume effects is shown or quoted in any given $\rho(\lambda, \delta)$. A Wilson-Dirac based overlap operator [31] with parameters $\rho=26 / 19$ and $r=1$ was used in all Dirac spectrum calculations.

Implicitly restarted Arnoldi method [32,33] was used to compute the eigenvalues and eigenvectors of the overlap operator. For all but one ensemble used in this study, it is efficient to first compute the eigenvalues of $D^{\dagger} D$ in a chiral sector, and then reconstruct the eigenvalues of $D$ using standard techniques [34]. For the $N=64 \mathrm{pgQCD}$ lattice at $T=1.12 T_{c}$, it becomes problematic to distinguish the eigenvalues of near-zero eigenmodes from those of exact zero modes. To ensure the reliability of numerical results in this case, we solved the eigenvalue problem for $D$ directly, utilizing a suitable polynomial spectral transformation to accelerate the convergence.

\section{APPENDIX D: ADDITIONAL DATA}

In this appendix we present additional lattice data to further support our conclusions.

A key to the proposed picture of thermal phases is the emergence of a remarkable separation of IR and UV physics at $T_{\mathrm{IR}}$. This signature aspect of the IR phase is reflected in sharp bimodality of Dirac spectral density and the resulting clear separation of scales (Sec. III). To convey this feature explicitly, we show in Fig. 4 spectral densities for both pgQCD and QCD in the IR phase. The data suggests the presence of dynamics in which IR and UV regimes act as separate independent "components" of the theory.

The $1 / \lambda$ behavior of $\rho(\lambda)$ over a wide IR range of scales can also be checked in a more direct manner, namely by the process of zooming in toward the infrared. In Fig. 5 we show this for $\mathrm{pgQCD}$ at $T=1.12 T_{c}$ on our largest lattice. With the lower spectral edge fixed at $0.1 \mathrm{MeV}$ to avoid finite volume effects, we plot $\rho(\lambda)$ up to 240,24 , and $2.4 \mathrm{MeV}$ respectively, with bin sizes correspondingly

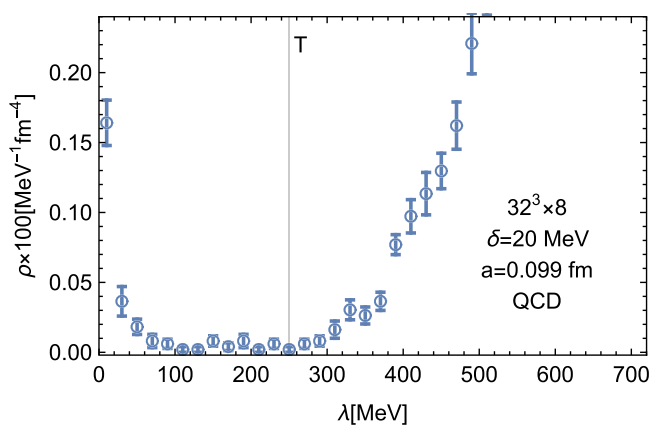

FIG. 4. The separation of IR and UV scales, manifested in a sharply bimodal overlap Dirac spectral density in pgQCD at $T=1.12 T_{c}$ (left) and QCD at $T=250 \mathrm{MeV}$. 

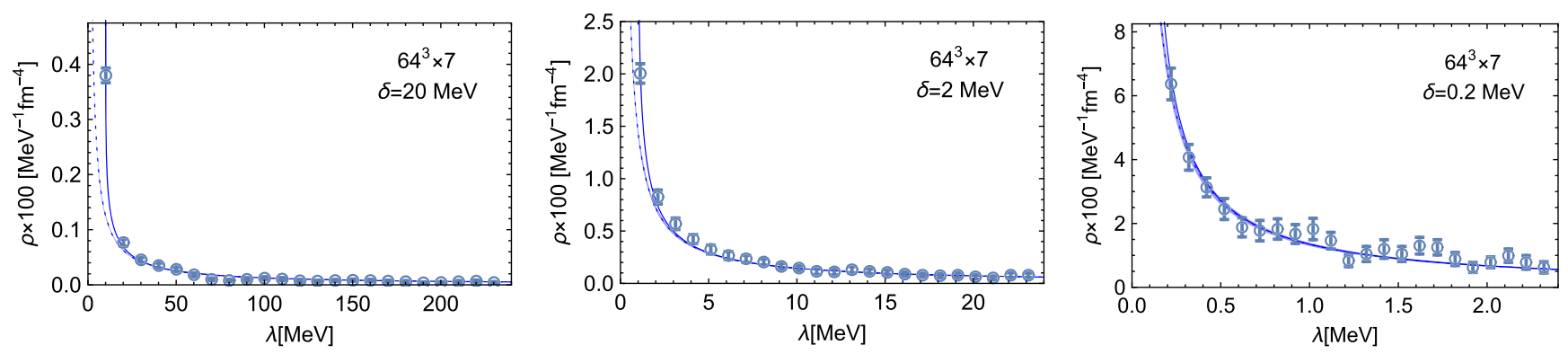

FIG. 5. Overlap Dirac spectral density for pgQCD in the IR phase $\left(T=1.12 T_{c}\right)$ over spectral ranges (and bin sizes) scaled by factors of 10 . Dashed lines represent direct $1 / \lambda$ fits, while the solid lines include the correction on finite bin size.
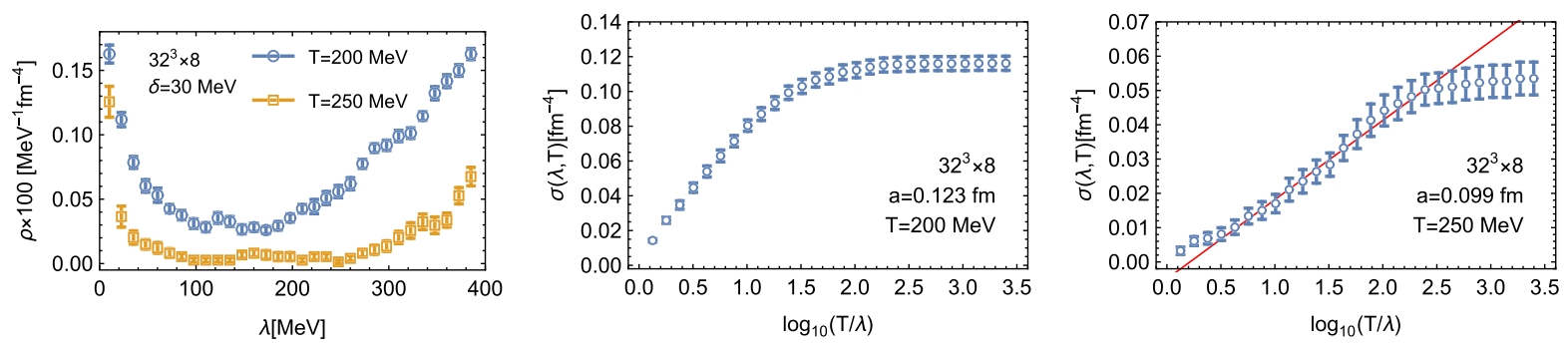

FIG. 6. Thermal transition to IR phase in QCD: $T_{\mathrm{IR}}$ is between $T=200 \mathrm{MeV}$ and $T=250 \mathrm{MeV}$.

scaled. In each case we fit the data to $1 / \lambda$ with bin size taken into account in the procedure (solid line) to avoid the finite bin distortion.

In addition to QCD data shown in Fig. 3 (bottom), we also computed Dirac spectra at $T=200 \mathrm{MeV}$. The relevant comparison to spectral behavior in the IR phase (analog of Fig. 3) is shown in Fig. 6. Note that $\sigma(x)$ is visibly approaching the linear regime that is characteristic of the IR phase. Together with the behavior of $\rho(\lambda)$ (left), this suggests that $T_{\mathrm{IR}}>200 \mathrm{MeV}$, thus leading to the estimate (2).

The scale $\Lambda_{\mathrm{IR}} \lesssim T$ marks the upper edge of the spectral region where $\rho(\lambda) \propto 1 / \lambda$ applies. For considerations of the thermodynamic limit, it is desirable to ask whether our data also provides a hint of lower edge $\Lambda_{\mathrm{IR}}^{\min }$, i.e., the point where the $1 / \lambda$ either softens up, or the negative power behavior entirely disappears. A convenient indicator of this

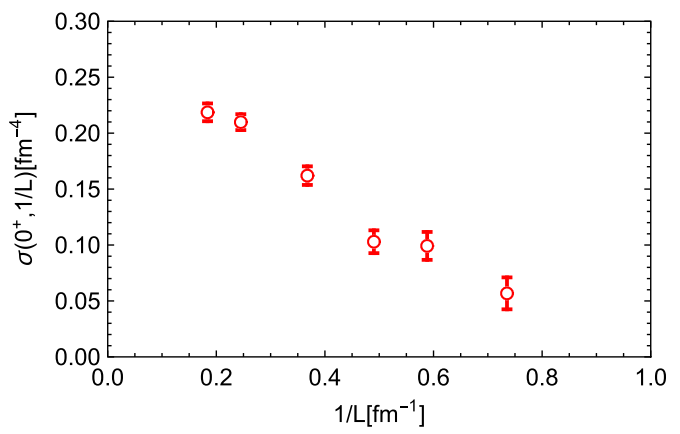

is $\sigma\left(0^{+}, 1 / L\right)$, namely the 4-volume density of nonzero Dirac modes smaller than the IR cutoff $1 / L$. Expressing the lower edge of the Dirac spectrum at finite $L$ as $\epsilon(L) / L$, we have

$$
\sigma\left(0^{+}, \frac{1}{L}\right)=\sigma\left(\frac{\epsilon(L)}{L}, \frac{1}{L}\right)=\int_{\epsilon(L) / L}^{1 / L} d \lambda \rho(\lambda)
$$

which vanishes in the $L \rightarrow \infty$ limit for all $p>-1$, where $\lambda^{p}$ is the leading IR behavior of $\rho(\lambda)$. Its $1 / L$ behavior in our pgQCD ensembles is shown in Fig. 7 (left). Since a turn toward zero for $1 / L \rightarrow 0$ is not observed, the available data does not suggest the existence of $\Lambda_{\mathrm{IR}}^{\min }$.

Since the proportionality constant $c$ of $1 / \lambda$ is stable with changing $L$ (see Fig. 2), and

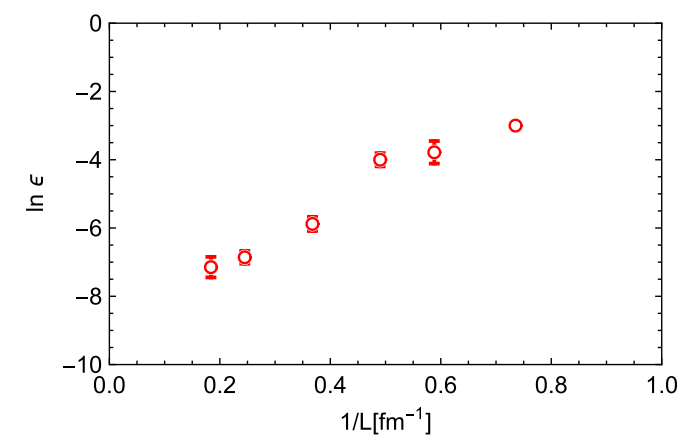

FIG. 7. Left: plot of $\sigma\left(0^{+}, 1 / L\right)$ for pgQCD ensembles. Right: plot of spectral edge parameter $\epsilon(L)$ (see text) for the same ensembles. 


$$
\begin{aligned}
\lim _{L \rightarrow \infty} \sigma\left(0^{+}, \frac{1}{L}\right) & \rightarrow-\lim _{L \rightarrow \infty} c(L) \ln \epsilon(L) \quad \text { for } \\
\rho(\lambda) & =\frac{c}{\lambda}, \quad \lambda>\frac{\epsilon}{L}
\end{aligned}
$$

the finite $\lim _{L \rightarrow \infty} \sigma\left(0^{+}, 1 / L\right)>0$, conveyed by Fig. 7 (left), implies finite $\lim _{L \rightarrow \infty} \epsilon(L)>0$. This is an important detail since $\epsilon(L)$ controls the IR edge of the spectrum. To check this explicitly, we estimate $\epsilon(L)$ directly using the relation

$$
\ln \epsilon(L)=-k \frac{\sigma\left(0^{+}, 1 / L\right)}{\sigma\left(e^{-k} / L, 1 / L\right)}
$$

which holds for arbitrary $k>0$ under the assumptions of (D2). The result for $k=3$, suitable for our range of $1 / L$ and the statistics, is shown in Fig. 7 (right), confirming the trend toward a small positive value of $\epsilon$ in the $L \rightarrow \infty$ limit.
[1] W. Busza, K. Rajagopal, and W. van der Schee, Annu. Rev. Nucl. Part. Sci. 68, 339 (2018).

[2] Y. Aoki, G. Endrodi, Z. Fodor, S. Katz, and K. Szabo, Nature (London) 443, 675 (2006).

[3] I. Arsene et al. (BRAHMS Collaboration), Nucl. Phys. A757, 1 (2005).

[4] B. B. Back et al. (PHOBOS Collaboration), Nucl. Phys. A757, 28 (2005).

[5] J. Adams et al. (STAR Collaboration), Nucl. Phys. A757, 102 (2005).

[6] K. Adcox et al. (PHENIX Collaboration), Nucl. Phys. A757, 184 (2005).

[7] B. Muller, J. Schukraft, and B. Wyslouch, Annu. Rev. Nucl. Part. Sci. 62, 361 (2012).

[8] G. Policastro, D. T. Son, and A. O. Starinets, Phys. Rev. Lett. 87, 081601 (2001).

[9] D. Gross and F. Wilczek, Phys. Rev. Lett. 30, 1343 (1973).

[10] H. Politzer, Phys. Rev. Lett. 30, 1346 (1973).

[11] B. Svetitsky and L. G. Yaffe, Nucl. Phys. B210, 423 (1982).

[12] A. Alexandru and I. Horváth, Phys. Lett. B 722, 160 (2013).

[13] A. Alexandru and I. Horváth, Nucl. Phys. B891, 1 (2015).

[14] A. Alexandru and I. Horváth, Phys. Rev. D 92, 045038 (2015).

[15] A. Patella, Phys. Rev. D 86, 025006 (2012).

[16] A. Cheng, A. Hasenfratz, G. Petropoulos, and D. Schaich, J. High Energy Phys. 07 (2013) 061.

[17] S. Borsanyi, Z. Fodor, C. Hoelbling, S. D. Katz, S. Krieg, and K. K. Szabo, Phys. Lett. B 730, 99 (2014).

[18] A. Bazavov et al. (HotQCD Collaboration), Phys. Rev. D 90, 094503 (2014).
[19] R. G. Edwards, U. M. Heller, J. E. Kiskis, and R. Narayanan, Phys. Rev. D 61, 074504 (2000).

[20] J. C. Osborn, D. Toublan, and J. J. M. Verbaarschot, Nucl. Phys. B540, 317 (1999).

[21] A. Alexandru and I. Horváth, AIP Conf. Proc. 1701, 030008 (2016).

[22] I. Horváth and A. Alexandru, Proc. Sci., LATTICE2015 (2015) 314 [arXiv:1511.09370].

[23] T. Banks and A. Zaks, Nucl. Phys. B196, 189 (1982).

[24] G. Mack, Commun. Math. Phys. 55, 1 (1977).

[25] C. Rohrhofer, Y. Aoki, G. Cossu, H. Fukaya, C. Gattringer, L. Ya. Glozman, S. Hashimoto, C. B. Lang, and S. Prelovsek, Phys. Rev. D 100, 014502 (2019).

[26] T. G. Kovacs and R. A. Vig, Phys. Rev. D 97, 014502 (2018).

[27] A. Tomiya, G. Cossu, S. Aoki, H. Fukaya, S. Hashimoto, T. Kaneko, and J. Noaki, Phys. Rev. D 96, 034509 (2017); 96, 079902(A) (2017).

[28] S. Sharma (HotQCD Collaboration), arXiv:1801.08500.

[29] S. Necco, Nucl. Phys. B683, 137 (2004).

[30] S. Borsanyi, Z. Fodor, C. Hoelbling, S. D. Katz, S. Krieg, C. Ratti, and K. K. Szabó (Wuppertal-Budapest Collaboration), J. High Energy Phys. 09 (2010) 073.

[31] H. Neuberger, Phys. Lett. B 417, 141 (1998).

[32] D. C. Sorensen, SIAM J. Matrix Anal. Appl. 13, 357 (1992).

[33] R. B. Lehoucq and D. C. Sorensen, SIAM J. Matrix Anal. Appl. 17, 789 (1996).

[34] A. Alexandru, M. Lujan, C. Pelissier, B. Gamari, and F. X. Lee, arXiv:1106.4964. 